\title{
Attractor of Beam Equation with Structural Damping under Nonlinear Boundary Conditions
}

\author{
Danxia Wang, ${ }^{1}$ Jianwen Zhang, ${ }^{1}$ Yinzhu Wang, ${ }^{2}$ and Sufang Zhang ${ }^{1}$ \\ ${ }^{1}$ Department of Mathematics, Taiyuan University of Technology, Taiyuan 030024, China \\ ${ }^{2}$ Department of Mathematics, Taiyuan University of Science and Technology, Taiyuan 030024, China \\ Correspondence should be addressed to Jianwen Zhang; jianwen.z2008@163.com
}

Received 12 December 2014; Revised 11 January 2015; Accepted 13 January 2015

Academic Editor: Mohamed Abd El Aziz

Copyright (c) 2015 Danxia Wang et al. This is an open access article distributed under the Creative Commons Attribution License, which permits unrestricted use, distribution, and reproduction in any medium, provided the original work is properly cited.

Simultaneously, considering the viscous effect of material, damping of medium, and rotational inertia, we study a kind of more general Kirchhoff-type extensible beam equation $u_{t t}-u_{x x t t}+u_{x x x x}-\sigma\left(\int_{0}^{l}\left(u_{x}\right)^{2} d x\right) u_{x x}-\phi\left(\int_{0}^{l}\left(u_{x}\right)^{2} d x\right) u_{x x t}=q(x)$, in $[0, L] \times R^{+}$ with the structural damping and the rotational inertia term. Little attention is paid to the longtime behavior of the beam equation under nonlinear boundary conditions. In this paper, under nonlinear boundary conditions, we prove not only the existence and uniqueness of global solutions by prior estimates combined with some inequality skills, but also the existence of a global attractor by the existence of an absorbing set and asymptotic compactness of corresponding solution semigroup. In addition, the same results also can be proved under the other nonlinear boundary conditions.

\section{Introduction}

In this paper, we will consider a kind of more general Kirchhoff-type beam equation. The physical origin of the problem lies in the theory of vibrations of an extensible beam of length $L$; moreover, during vibration, the elements of a beam not only perform a translatory motion but also rotate.

A mathematical model for this problem is an initial boundary value problem for the nonlinear Kirchhoff-type beam equation

$$
\begin{aligned}
u_{t t} & -u_{x x t t}+u_{x x x x}-\sigma\left(\int_{0}^{L}\left(u_{x}\right)^{2} d x\right) u_{x x} \\
& -\phi\left(\int_{0}^{L}\left(u_{x}\right)^{2} d x\right) u_{x x t} \\
= & q(x), \quad(x, t) \in(0, L) \times R^{+} .
\end{aligned}
$$

We assume the nonlinear boundary conditions

$$
\begin{gathered}
u(0, t)=u_{x}(L, t)=u_{x x}(0, t)=0, \\
u_{x x x}(L, t)=f(u(L, t))+g\left(u_{t}(L, t)\right)
\end{gathered}
$$

and the initial conditions

$$
u(x, 0)=u^{0}(x), \quad u_{t}(x, 0)=u^{1}(x), \quad x \in(0, L) .
$$

Here the unknown function $u(x, t)$ is the elevation of the surface of beam, $u^{0}(x)$ and $u^{1}(x)$ are the given initial value functions, and the subscript $t$ and $x$ denote derivative with respect to $t$ and $x$, respectively. $-u_{x x t t}$ expresses the rotational inertia, and nonlinear terms $\sigma\left(\int_{0}^{L}\left(u_{x}\right)^{2} d x\right) u_{x x}$ and $\phi\left(\int_{0}^{L}\left(u_{x}\right)^{2} d x\right) u_{x x t}$ represent the extensibility effects and the structural damping, respectively. $q(x)$ is a static load. Moreover the assumptions on nonlinear functions $\sigma(\cdot), \phi(\cdot)$, $g(\cdot)$, and $f(\cdot)$ and the external force function $q(x)$ will be specified later.

In (1), when the structural damping term and the rotational inertia term are absent, (1) is a model for vibrations of tensible beam. This was proposed by Woinowsky-Krieger [1] in the form

$$
u_{t t}+u_{x x x x}-\left(\alpha+\beta \int_{0}^{L} u_{x}^{2} d x\right) u_{x x}=0 .
$$


One of the first mathematical analyses for equation

$$
u_{t t}+u_{x x x x}-M\left(\int_{0}^{L}\left(u_{x}\right)^{2} d x\right) u_{x x}=0
$$

was done by Ball [2] which was later extended to an abstract setting by defining a linear operator $A$ by Medeiros [3]. In [4], Patcheu obtained the decay of the energy for above equation when a nonlinear damping $g\left(u_{t}\right)$ was effective in $\Omega$. In addition, the attractor on extensible beams with null boundary conditions was considered by several authors. We quote, for instance, [5-7], and so on. But the longtime behavior of the beam equation with nonlinear boundary conditions was paid little attention. We also refer the reader to a few works. One of the first studies in this direction was done by Pazoto and Menzala [8], where stabilization of a thermoelastic extensible beam was considered. Motivated by the result, Ma proved the existence of global solutions and the existence of a global attractor in [9] and [10], respectively, for the Kirchhoff-type beam equation

$$
u_{t t}+u_{x x x x}-M\left(\int_{0}^{L}\left(u_{x}\right)^{2} d x\right) u_{x x}=0
$$

with the absence of the structural damping and the rotational inertia, subjected to the nonlinear boundary conditions

$$
\begin{gathered}
u(0, t)=u_{x}(0, t)=u_{x x}(L, t)=0, \\
u_{x x x}(L, t)-M\left(\int_{0}^{L}\left(u_{x}\right)^{2} d x\right) u_{x}(L, t) \\
=f(u(L, t))+g\left(u_{t}(L, t)\right) .
\end{gathered}
$$

In the following, we mentioned some results on longtime behavior of beam equation with the rotational inertia term. Under null boundary conditions, Geredeli and Lasiecka [11] considered the existence of a compact attractor of beam

$$
\begin{aligned}
u_{t t} & -\alpha \Delta u_{t t}+\Delta^{2} u+d(x) g\left(u_{t}\right) \\
& -\alpha \operatorname{div}(d(x)) g\left(\nabla u_{t}\right)-F(u)=p(x)
\end{aligned}
$$

with a rotational inertia term. Under nonlinear boundary conditions

$$
u=0, \quad \Delta u=-g\left(\frac{\partial u_{t}}{\partial v}\right)
$$

Ji and Lasiecka [12] considered the semilinear Kirchhoff equation

$$
u_{t t}-\gamma \Delta u_{t t}+\Delta^{2} u+f(u)=0
$$

with rotational inertia, and they showed that the above problem is uniformly stabilized with uniform energy decay rates.

In addition, we also mentioned some results on longtime behavior of the equation with the structural damping term. Chueshov [13] studied the global attractor with a structural damping of the form $\sigma\left(\|\nabla u\|^{2}\right)(-\Delta)^{\theta} u_{t}$ with $1 / 2 \leq \theta \leq 1$.
Chueshov [14] and Yang et al. [15] considered the global attractor for the Kirchhoff-type equation

$$
u_{t t}-\sigma\left(\|\nabla u\|^{2}\right) \Delta u_{t}-\phi\left(\|\nabla u\|^{2}\right) \Delta u+f(u)=h(x)
$$

with structural damping under null boundary conditions, respectively.

On (1) under the following other nonlinear boundary conditions

$$
\begin{gathered}
u(L, t)=u_{x}(0, t)=u_{x x}(L, t)=0, \\
u_{x x x}(0, t)=f(u(0, t))+g\left(u_{t}(0, t)\right)
\end{gathered}
$$

we also can get the same on the existence of global solutions and the existence of global attractor.

Our fundamental assumptions on $\sigma(\cdot), \phi(\cdot), g(\cdot), f(\cdot)$, and $q(x)$ are given as follows.

Assumption 1. We assume that $\sigma(\cdot), \phi(\cdot) \in C^{1}(R)$ are all nondecreasing and satisfy

$$
\sigma(z) z \geq \widehat{\sigma}(z) \geq 0, \quad \forall z \geq 0,
$$

where $\widehat{\sigma}(z)=\int_{0}^{z} \sigma(s) d s \geq 0$. Moreover

$$
\begin{gathered}
\sigma(0)=0, \quad \phi(0)=0, \\
\sigma(s) \geq \phi(s) \geq \alpha+\beta s^{\gamma} \quad(\alpha, \beta>0, \gamma \geq 1), \forall s \in R^{+} .
\end{gathered}
$$

Assumption 2. The function $g(\cdot): R \rightarrow R$ is of class $C^{1}(R)$ and satisfies $g(0)=0$, and there exist constants $k_{1}, k_{2}$ and $r \geq 0$ such that

$$
\begin{gathered}
(g(u)-g(v))(u-v) \geq k_{1}|u-v|^{2}, \quad \forall u, v \in R, \\
|g(u)-g(v)| \leq k_{2}\left(1+|u|^{r}+|v|^{r}\right)|u-v|, \quad \forall u, v \in R .
\end{gathered}
$$

Assumption 3. The function $f(\cdot): R \rightarrow R$ is of class $C^{1}(R)$ and satisfies $f(0)=0$, and there exist constants $k$ and $\varrho \geq 0$ such that

$$
\begin{aligned}
|f(u)-f(v)| \leq k\left(1+|u|^{\varrho}+|v|^{\varrho}\right)|u-v|, \quad \forall u, v \in R \\
-L_{0} \leq \widehat{f}(u) \leq f(u) u+L_{1},
\end{aligned}
$$

where $\widehat{f}(z)=\int_{0}^{z} f(s) d s \geq 0$.

Assumption 4. Consider $q(x) \in L^{2}(0, L)$.

Under the above assumptions, we prove the existence of global solutions and the existence of a global attractor of extensible beam equation system (1)-(4). And the paper is organized as follows. In Section 2, we introduce some Sobolev spaces. In Section 3, we discuss the existence of global strong and weak solutions. In Section 4, we establish the result of the existence of a global attractor in $V \times U$. 


\section{Preliminaries}

Our analysis is based on the following Sobolev spaces. Let

$$
\begin{gathered}
U=\left\{u \in H^{1}(0, L) ; u(0)=0\right\}, \\
V=\left\{u \in H^{2}(0, L) ; u(0)=u_{x}(L)=0\right\}, \\
W=\left\{u \in H^{4}(0, L) \cap V ; u_{x x}=0\right\} .
\end{gathered}
$$

Motivated by the boundary condition (3) we assume, for regular solutions, that data $\left(u^{0}, u^{1}\right)$ satisfies the following compatibility condition:

$$
u_{x x x}^{0}(L)=f\left(u^{0}(L)\right)+g\left(u^{1}(L)\right)
$$

Then for regular solutions we consider the phase space

$$
H_{1}=\left\{\left(u^{0}, u^{1}\right) \in W \times W \text {; satisfies condition (22) }\right\} .
$$

In the case of weak solutions we consider the phase space

$$
H_{0}=V \times U=\overline{H_{1}},
$$

which guarantees that, for regular data, the nonlinear condition (22) holds. In $H_{0}$ we adopt the norm defined by

$$
\|u, v\|_{H_{0}}^{2}=\|(u, v)\|_{V \times U}^{2}=\left\|u_{x x}\right\|^{2}+\left\|v_{x}\right\|^{2} .
$$

\section{The Existence of Global Solutions}

Firstly, using the classical Galerkin method, we can establish the existence and uniqueness of regular solution to problem (1)-(4). We state it as follows.

Theorem 1. Assume Assumptions 1-4 and (22)-(23) hold, for any initial data $\left(u^{0}, u^{1}\right) \in H_{1}$; then problem (1)-(4) has a unique regular solution $u(t)$ with

$$
u \in L_{\mathrm{loc}}^{\infty}\left(R^{+}, W\right) \cap C^{0}([0, \infty) ; V) \cap C^{1}([0, \infty) ; U) .
$$

Moreover,

$$
\left\|u_{t}\right\|^{2}+\left\|u_{x t}\right\|^{2}+\left\|u_{x x}\right\|^{2}+\widehat{\sigma}\left(\left\|u_{x}\right\|^{2}\right) \leq M_{1},
$$

where $M_{1}>0$ depends on the initial data and $q$, but not on $t>0$.

Proof. Let us consider the variational problem associated with (1)-(4): find $u(t) \in W$ such that

$$
\begin{aligned}
& \int_{0}^{L} u_{t t} \omega d x+\int_{0}^{L} u_{x t t} \omega_{x} d x+\int_{0}^{L} u_{x x} \omega_{x x} d x \\
& \quad+\sigma\left(\left\|u_{x}\right\|^{2}\right) \int_{0}^{L} u_{x} \omega_{x} d x+\phi\left(\left\|u_{x}\right\|^{2}\right) \int_{0}^{L} u_{x t} \omega_{x} d x \\
& \quad+f(u(L, t)) \omega(L)+g\left(u_{t}(L, t)\right) \omega(L) \\
& =\int_{0}^{L} q(x) \omega d x
\end{aligned}
$$

for all $\omega \in V$. This is done with the Galerkin approximation methods which is standard. Here we denote the approximate solution by $u^{m}(t)$. We can get the theorem by proving the existence of approximation solution, the estimate of approximation solution, convergence, uniqueness, and $u \in C^{0}([0, \infty) ; V) \cap C^{1}([0, \infty) ; U)$. In the following we give the estimates of approximation solution, the proof of uniqueness of solution, and the proof of $u \in C^{0}([0, \infty) ; V) \cap$ $C^{1}([0, \infty) ; U)$.

Estimate 1. In approximate equation of (28), putting $\omega=u_{t}^{m}(t)$ and considering $\widehat{\sigma}(z)=\int_{0}^{z} \sigma(s) d s, \widehat{f}(z)=\int_{0}^{z} f(s) d s$, using Schwarz inequality, and then integrating from 0 to $t<t_{m}$, we see that

$$
\begin{aligned}
& \left\|u_{t}^{m}\right\|^{2}+\left\|u_{x t}^{m}\right\|^{2}+\left\|u_{x x}^{m}\right\|^{2}+\widehat{\sigma}\left(\left\|u_{x}^{m}\right\|^{2}\right) \\
& +\widehat{f}\left(u^{m}(l, t)\right)+2 \int_{0}^{t} \phi\left(\left\|u_{x}^{m}\right\|^{2}\right)\left\|u_{x t}^{m}\right\|^{2} d s \\
& \quad+2 \int_{0}^{t} g\left(u_{t}^{m}(l, t)\right) u_{t}^{m}(l, t) d s \\
& \leq \int_{0}^{t}\|q(x)\|^{2} d s+\int_{0}^{t}\left\|u_{t}^{m}\right\|^{2} d s+\left\|u_{t}^{m}(0)\right\|^{2} \\
& \quad+\left\|u_{x t}^{m}(0)\right\|^{2}+\left\|u_{x x}^{m}(0)\right\|^{2}+\widehat{\sigma}\left(\left\|u_{x}^{m}(0)\right\|^{2}\right) \\
& \quad+\widehat{f}\left(u^{m}(l, 0)\right) .
\end{aligned}
$$

Taking into account the assumptions $\int_{0}^{t} g\left(u_{t}^{m}(l, t)\right) u_{t}^{m}(l, t) d s \geq$ $0, \phi(\cdot) \geq 0$, and $\widehat{f}, \widehat{\sigma} \geq 0$ of $f, g, \sigma$, and $\phi$, we see that there exists $M_{1}>0$ such that

$$
\left\|u_{t}^{m}\right\|^{2}+\left\|u_{x t}^{m}\right\|^{2}+\left\|u_{x x}^{m}\right\|^{2}+\widehat{\sigma}\left(\left\|u_{x}^{m}\right\|^{2}\right) \leq M_{1}
$$

for all $t \in[0, T]$ and for all $m \in N$.

Estimate 2. In approximate equation of (28), integrating by parts with $\omega=u_{t t}^{m}(0)$ and $t=0$ and considering the compatibility condition (22) and then using Schwarz inequality and the mean value inequality, we see that there exists $M_{2}>0$ such that

$$
\left\|u_{t t}^{m}(0)\right\|^{2}+\left\|u_{x t t}^{m}(0)\right\|^{2} \leq M_{2}
$$

for all $t \in[0, T]$ and for all $m \in N$.

Estimate 3. Let us fix $t, \xi>0$ such that $\xi<T-t$. Taking the difference of approximate equation of (28) with $t=t+\xi$ and $t=t$ and replacing $\omega$ by $u_{t}^{m}(t+\xi)-u_{t}^{m}(t)$, we can find a constant $M_{3}>0$, depending only on $T$, such that

$$
\left\|u_{t t}^{m}\right\|^{2}+\left\|u_{x t t}^{m}\right\|^{2}+\left\|u_{x x t}^{m}\right\|^{2} \leq M_{3}, \quad \forall m \in N, \forall t \in[0, T] .
$$

Uniqueness. Let $u, v$ be two solutions of (1)-(4) with the same initial data. Then writing $z=u-v$ and taking the difference (28) with $u=u$ and $u=v$ and replacing $\omega$ by $z_{t}$ and 
then using mean value theorem and the Young inequalities combined with Estimates 1 and 3, we deduce that, for some constant $C>0$,

$$
\begin{aligned}
& \frac{d}{d t}\left(\left\|z_{t}^{m}\right\|^{2}+\left\|z_{x t}^{m}\right\|^{2}+\left\|z_{x x}^{m}\right\|^{2}\right) \\
& \quad \leq C\left(\left\|z_{t}^{m}\right\|^{2}+\left\|z_{x t}^{m}\right\|^{2}+\left\|z_{x x}^{m}\right\|^{2}\right), \quad \forall t \in(0, T) .
\end{aligned}
$$

Then from Gronwall's lemma we see that $u=v$.

The Proof of $u \in C^{0}([0, \infty) ; V) \cap C^{1}([0, \infty) ; U)$. Since $u_{x x}, u_{x x t} \in L^{2}\left(0, \infty ; L^{2}(0, L)\right)$, we get $u(x, t) \in C^{0}([0, \infty) ; V)$. Similarly, $u(x, t) \in C^{1}([0, \infty) ; U)$. The proof of Theorem 1 is completed.

Theorem 2. Assume the assumptions of Theorem 1 and (24) hold; if the initial data $\left(u^{0}, u^{1}\right) \in H_{0}$, then there exists a unique weak solution $u(t)$ of problem (1)-(4) which depends continuously on initial data with respect to the norm of $V \times U$.

Proof. Let us consider $\left\{u^{0}, u^{1}\right\} \in \overline{W \times W}=V \times L^{2}$, and since $W \times W$ is dense in $V \times U$, there exists $\left\{u_{\mu}^{0}, u_{\mu}^{1}\right\} \subset W \times W$, such that

$$
\begin{aligned}
& u_{\mu}^{0} \rightarrow u^{0}, \quad \text { in } V ; \\
& u_{\mu}^{1} \longrightarrow u^{1}, \quad \text { in } U .
\end{aligned}
$$

We observe that, for each $\mu \in N$, there exists $u_{\mu}$, smooth solution of the initial boundary value problem (1)-(4) which satisfies

$$
\begin{gathered}
u_{t t \mu}-u_{x x t \mu}+u_{x x x x \mu}-\sigma\left(\int_{0}^{L}\left(u_{x \mu}\right)^{2} d x\right) u_{x x \mu} \\
-\phi\left(\int_{0}^{L}\left(u_{x \mu}\right)^{2} d x\right) u_{x x t \mu}=q(x), \quad(x, t) \in(0, L) \times R^{+}, \\
u_{\mu}(x, 0)=u_{\mu}^{0}(x), \quad u_{t \mu}(x, 0)=u_{\mu}^{1}(x), \quad x \in(0, L), \\
u_{\mu}(0, t)=u_{x \mu}(L, t)=u_{x x \mu}(0, t)=0, \quad t \in(0, \infty), \\
u_{x x x \mu}(L, t)=f\left(u_{\mu}(L, t)\right)+g\left(u_{t \mu}(L, t)\right), \quad t \in(0, \infty) .
\end{gathered}
$$

Considering the arguments used in the estimate of the existence of solution, we obtain

$$
\left\|u_{t \mu}\right\|^{2}+\left\|u_{x t \mu}\right\|^{2}+\left\|u_{x x \mu}\right\|^{2} \leq C_{0}
$$

where $C_{0}$ is a positive constant independent of $\mu \in N$.

Defining $Z_{\mu, \sigma}=u_{\mu}-u_{\sigma}: \mu, \sigma \in N$ where $u_{\mu}$ and $u_{\sigma}$ are regular solutions of (35), following the steps already used in the uniqueness of regular solution for (1)-(4) and considering the convergence given in (34) $\left(u_{\mu}^{0} \rightarrow u^{0}\right.$ in $V$ and $u_{\mu}^{1} \rightarrow$ $u^{1}$ in $\left.U\right)$, we deduce that there exists $u$ such that

$$
\begin{gathered}
u_{\mu} \longrightarrow u \quad \text { strongly in } C([0, T) ; V), \\
u_{t \mu} \longrightarrow u_{t} \quad \text { strongly in } C([0, T) ; U) .
\end{gathered}
$$

From the above convergence, we can pass to the limit using standard arguments in order to obtain

$$
\begin{aligned}
u_{t t}- & u_{x x t t}+u_{x x x x}-\sigma\left(\int_{0}^{L}\left(u_{x}\right)^{2} d x\right) u_{x x} \\
& -\phi\left(\int_{0}^{L}\left(u_{x}\right)^{2} d x\right) u_{x x t} \\
= & q(x), \quad(x, t) \in(0, L) \times R^{+}, \\
u(x, 0)= & u^{0}(x), \quad u_{t}(x, 0)=u^{1}(x), \quad x \in(0, L) .
\end{aligned}
$$

Theorem 2 is proved.

Remark 3. Theorem 2 implies that problem (1)-(4) defines a nonlinear $C_{0}$-semigroup $S(t)$ on $H_{0}$. Indeed, let us set $S(t)\left(u^{0}, u^{1}\right)=\left(u(t), u_{t}(t)\right)$, where $u$ is the unique solution corresponding to initial data $\left(u^{0}, u^{1}\right) \in H_{0}$. Moreover, the operator $S(t)$ defined in $H_{0}$ maps $H_{0}$ into itself and it enjoys the usual semigroup properties

$$
\begin{gathered}
S(t+\tau)=S(t)+S(\tau), \quad \forall t, \tau \in R, \\
S(0)=I .
\end{gathered}
$$

And it is obvious that the map $\{S(t), t \geq 0\}$ is continuous in space $H_{0}$.

\section{The Existence of Global Attractor}

In this section, we give the existence of a global attractor.

A global attractor for a $C_{0}$-semigroup $S(t)$ defined on a complete metric space $H$ is a bounded closed subset $\mathbb{A} \subset H$ which is positive fully invariant, that is, $S(t) \mathbb{A}=\mathbb{A}$, for all $t \geq 0$, and uniformly attracting, that is,

$$
\operatorname{dist}(S(t) B, \mathbb{A})=\sup _{x \in S(t) B} \inf _{y \in \mathbb{A}} d(x, y) \longrightarrow 0 \quad \text { as } t \longrightarrow \infty \text {, }
$$

for any bounded set $B \subset H$.

A bounded set $\mathbb{B} \subset H$ is an absorbing set for $S(t)$ if, for any bounded set $B \subset H$, there exists $t_{B}=t(B) \geq 0$ such that

$$
S(t) B \subset \mathbb{B}, \quad \forall t \geq t_{B}
$$

which defines $(H, S(t))$ as a dissipative dynamical system.

Theorem 4. Assume the hypotheses of Theorem 2 and $\varrho=$ $r=0, k>0$ is sufficiently small, and then the corresponding semigroup $S(t)$ of problem (1)-(4) has an absorbing set in $\mathrm{H}_{0}$.

Proof. Now, we show that semigroup $S(t)$ has an absorbing set in $H_{0}$. Firstly, we can calculate the total energy functional

$$
\begin{aligned}
E(t)= & \frac{1}{2}\left(\left\|u_{t}\right\|^{2}+\left\|u_{x t}\right\|^{2}+\left\|u_{x x}\right\|^{2}+\widehat{\sigma}\left(\left\|u_{x}\right\|^{2}\right)\right) \\
& +\widehat{f}(u(L, t))-\int_{0}^{L} q u(t) d x .
\end{aligned}
$$


Let us fix an arbitrary bounded set $B \subset H_{0}$ and consider the solutions of problem (1)-(4) given by $\left(u(t), u_{t}(t)\right)=$ $S(t)\left(u^{0}, u^{1}\right)$ with $\left(u^{0}, u^{1}\right) \in B$. Our analysis is based on the modified energy functional

$$
\widetilde{E}(t)=E(t)+L^{4}\|q\|^{2}+L_{0}
$$

It is easy to see that $(d / d t) E(t)=(d / d t) \widetilde{E}(t)$.

Indeed, since $u(0)=0$, we have

$$
\begin{aligned}
|u(L, t)|^{2} & =|u(L, t)-u(0, t)|^{2} \\
& =\left|\int_{0}^{L} u_{x} d x\right|^{2} \\
& \leq \int_{0}^{L} d x \int_{0}^{L} u_{x}^{2} d x=L\left\|u_{x}\right\|^{2} .
\end{aligned}
$$

In a similar way, since $u(0, t)=u_{x}(L, t)=u_{x x}(0, t)=0$, the following inequalities hold

$$
\begin{gathered}
\|u\| \leq \sqrt{L}\left\|u_{x}\right\|, \quad\left\|u_{x}\right\| \leq L\left\|u_{x x}\right\|, \\
\|u\| \leq L^{2}\left\|u_{x x}\right\| .
\end{gathered}
$$

Now let us define

$$
\varphi(t)=\int_{0}^{L}\left(u_{t}-u_{x x t}\right) u d x
$$

By multiplying $(1)$ by $u_{t}$ and integrating over $(0, L)$, we have

$$
\frac{d}{d t} E(t)+\phi\left(\left\|u_{x}\right\|^{2}\right)\left\|u_{x t}\right\|^{2}+g\left(u_{t}(l, t)\right) u_{t}(l, t)=0 .
$$

Then, multiplying (1) by $u$ and integrating over $(0, L)$, we obtain

$$
\begin{aligned}
& \frac{d}{d t} \varphi(t)-\left\|u_{t}\right\|^{2}-\left\|u_{x t}\right\|^{2}+\left\|u_{x x}\right\|^{2}+u(L) u_{x x x}(L) \\
& \quad+\sigma\left(\left\|u_{x}\right\|^{2}\right)\left\|u_{x}\right\|^{2}+\phi\left(\left\|u_{x}\right\|^{2}\right) \int_{0}^{L} u_{x} u_{x t} d x=\int_{0}^{L} q u d x .
\end{aligned}
$$

Taking into account the boundary condition (3), we get

$$
\begin{aligned}
& \frac{d}{d t} \varphi(t)-\left\|u_{t}\right\|^{2}-\left\|u_{x t}\right\|^{2}+\left\|u_{x x}\right\|^{2} \\
& \quad+u(L)\left[f(u(L, t))+g\left(u_{t}(L, t)\right)\right] \\
& \quad+\sigma\left(\left\|u_{x}\right\|^{2}\right)\left\|u_{x}\right\|^{2}+\phi\left(\left\|u_{x}\right\|^{2}\right) \int_{0}^{L} u_{x} u_{x t} d x \\
& =\int_{0}^{L} q u d x .
\end{aligned}
$$

Taking the sum of (47) with $\varepsilon$ times (49) and using the Schwarz inequality, we have

$$
\begin{aligned}
& \frac{d}{d t} E(t)+\varepsilon \frac{d}{d t} \varphi(t)+\varepsilon E(t) \\
& \quad+\frac{\varepsilon}{2}\left\|u_{t}\right\|^{2}+\frac{\varepsilon}{2}\left\|u_{x t}\right\|^{2}+\frac{\varepsilon}{2}\left\|u_{x x}\right\|^{2} \\
& \quad+\varepsilon \sigma\left(\left\|u_{x}\right\|^{2}\right)\left\|u_{x}\right\|^{2}-\frac{\varepsilon}{2} \widehat{\sigma}\left(\left\|u_{x}\right\|^{2}\right) \\
& \quad+\phi\left(\left\|u_{x}\right\|^{2}\right)\left\|u_{x t}\right\|^{2}+g\left(u_{t}(L, t)\right) u_{t}(L, t) \\
& =2 \varepsilon\left(\left\|u_{t}\right\|^{2}+\left\|u_{x t}\right\|^{2}\right)-\varepsilon \phi\left(\left\|u_{x}\right\|^{2}\right) \int_{0}^{L} u_{x} u_{x t} d x \\
& \quad+\varepsilon \widehat{f}(u(L, t))-\varepsilon f(u(L, t)) u(L, t) \\
& \quad-\varepsilon g\left(u_{t}(L, t)\right) u(L, t) .
\end{aligned}
$$

So, using (15), we obtain

$$
g\left(u_{t}(L, t)\right) u_{t}(L, t) \geq k_{1}\left|u_{t}(L, t)\right|^{2}
$$

and using (16) and (44), we obtain

$$
\begin{aligned}
\varepsilon g\left(u_{t}(L, t)\right) u(L, t) & \leq 3 \varepsilon k_{2}\left|u_{t}(L, t)\right||u(L, t)| \\
& \leq 3 L^{3 / 2} \varepsilon k_{2} \varepsilon\left\|u_{x x}\right\|\left|u_{t}(L, t)\right| \\
& \leq 9 \varepsilon k_{2}^{2} L^{3}\left|u_{t}(L, t)\right|^{2}+\frac{\varepsilon}{4}\left\|u_{x x}\right\|^{2} .
\end{aligned}
$$

Taking into account (18) and (44)-(45), we have

$$
\begin{aligned}
\mid \varepsilon \widehat{f} & (u(L, t))-\varepsilon f(u(L, t)) u(L, t) \mid \\
& \leq\left|\frac{\varepsilon}{2} f(u(L, t)) u(L, t)\right|+\varepsilon L_{1} \\
& \leq \frac{\varepsilon}{2} 3 k\|u\|_{\infty}^{2}+\varepsilon L_{1} \\
& \leq \frac{\varepsilon}{2} 3 k L^{3}\left\|u_{x x}\right\|^{2}+\varepsilon L_{1} .
\end{aligned}
$$

Using the mean value inequality, we get

$$
\begin{aligned}
& \left|-\varepsilon \phi\left(\left\|u_{x}\right\|^{2}\right) \int_{0}^{L} u_{x} u_{x t} d x\right| \\
& \leq \frac{\varepsilon}{2} \phi\left(\left\|u_{x}\right\|^{2}\right)\left(\left\|u_{x}\right\|^{2}+\left\|u_{x t}\right\|^{2}\right) .
\end{aligned}
$$


Then inserting (51)-(54) into (50), we obtain

$$
\begin{aligned}
& \frac{d}{d t} E(t)+\varepsilon \frac{d}{d t} \varphi(t)+\varepsilon E(t)+\frac{\varepsilon}{2}\left\|u_{t}\right\|^{2} \\
& \quad+\frac{\varepsilon}{2}\left\|u_{x t}\right\|^{2}+\frac{\varepsilon}{2}\left\|u_{x x}\right\|^{2} \\
& \quad+\varepsilon \sigma\left(\left\|u_{x}\right\|^{2}\right)\left\|u_{x}\right\|^{2}-\frac{\varepsilon}{2} \widehat{\sigma}\left(\left\|u_{x}\right\|^{2}\right) \\
& \quad+\phi\left(\left\|u_{x}\right\|^{2}\right)\left\|u_{x t}\right\|^{2}+k_{1}\left|u_{t}(L)\right|^{2} \\
& \leq 2 \varepsilon\left(\left\|u_{t}\right\|^{2}+\left\|u_{x t}\right\|^{2}\right)+\frac{\varepsilon}{2} \phi\left(\left\|u_{x}\right\|^{2}\right)\left\|u_{x t}\right\|^{2} \\
& \quad+\frac{\varepsilon}{2} \phi\left(\left\|u_{x}\right\|^{2}\right)\left\|u_{x}\right\|^{2} \\
& \quad+\varepsilon 3 k L^{3}\left\|u_{x x}\right\|^{2}+\varepsilon L_{1}+9 \varepsilon k_{2}^{2} L^{3}\left|u_{t}(L, t)\right|^{2}+\frac{\varepsilon}{4}\left\|u_{x x}\right\|^{2} .
\end{aligned}
$$

Taking $0<\varepsilon \leq \min \left\{k_{1} / k_{2}^{2} L^{3}, 1\right\}=\varepsilon_{0}$ small enough, we get

$$
k_{1}\left|u_{t}(L, t)\right|^{2}>9 \varepsilon k_{2}^{2} L^{3}\left|u_{t}(L, t)\right|^{2} .
$$

Since with $k>0$ sufficiently small we have $1-6 k L^{3} \geq 0$, we get

$$
\frac{\varepsilon}{2}\left\|u_{x x}\right\|^{2} \geq \frac{1}{2} \varepsilon 3 k L^{3}\left\|u_{x x}\right\|^{2}+\frac{\varepsilon}{4}\left\|u_{x x}\right\|^{2} .
$$

Using $\phi(s) \geq \alpha+\beta s^{\gamma},(\gamma \geq 1$, for all $s \in R)$, we get

$$
\begin{aligned}
\frac{1}{2} \phi\left(\left\|u_{x}\right\|^{2}\right)\left\|u_{x t}\right\|^{2} & \geq \frac{\alpha}{2}\left\|u_{x t}\right\|^{2}+\frac{\beta}{2}\left\|u_{x}\right\|^{2 \gamma}\left\|u_{x t}\right\|^{2} \\
& \geq \frac{\alpha}{2}\left\|u_{x t}\right\|^{2} \\
& \geq \frac{\alpha}{4 \sqrt{L}}\left\|u_{t}\right\|^{2}+\frac{\alpha}{4}\left\|u_{x t}\right\|^{2} .
\end{aligned}
$$

So setting $0<\varepsilon \leq \min \left\{\varepsilon_{0}, \alpha / 8 \sqrt{L}\right\}$ small enough,

$$
\frac{\alpha}{4 \sqrt{L}}\left\|u_{t}\right\|^{2}+\frac{\alpha}{4}\left\|u_{x t}\right\|^{2} \geq 2 \varepsilon\left(\left\|u_{t}\right\|^{2}+\left\|u_{x t}\right\|^{2}\right) .
$$

Considering that $\sigma(z) z-\widehat{\sigma}(z) \geq 0$ and $\sigma(s) \geq \phi(s)$, for all $s \in R$, we obtain

$$
\varepsilon \sigma\left(\left\|u_{x}\right\|^{2}\right)\left\|u_{x}\right\|^{2}-\frac{\varepsilon}{2} \widehat{\sigma}\left(\left\|u_{x}\right\|^{2}\right)-\frac{\varepsilon}{2} \phi\left(\left\|u_{x}\right\|^{2}\right)\left\|u_{x}\right\|^{2} \geq 0 .
$$

Therefore with (56)-(60), (55) is transformed into

$$
\frac{d}{d t} E(t)+\varepsilon \frac{d}{d t} \varphi(t)+\varepsilon E(t) \leq \varepsilon L_{1}
$$

Adding $\varepsilon L_{0}+\varepsilon L^{4}\|h\|^{2}$ on both sides of inequality (61) and taking into account that $(d / d t) E(t)=(d / d t) \widetilde{E}(t)$, we obtain

$$
\frac{d}{d t}(\widetilde{E}(t)+\varepsilon \varphi(t))+\varepsilon \widetilde{E}(t) \leq \varepsilon\left(L_{0}+L_{1}+L^{4}\|q\|^{2}\right) .
$$

Now, let us set

$$
\widetilde{E}_{\varepsilon}(t)=\widetilde{E}(t)+\varepsilon \varphi(t) .
$$

Then since from (45)- $\int_{0}^{L} q u d x \geq-L^{2}\|q\|\left\|u_{x x}\right\| \geq-L^{4}\|q\|^{2}-$ $(1 / 4)\left\|u_{x x}\right\|^{2}$, also $\widehat{f}(u(L, t)) \geq-L_{0}$ and $\widehat{\sigma}\left(\left\|u_{x}\right\|^{2}\right) \geq 0$, we can get

$$
\begin{aligned}
\widetilde{E}(t) & \geq \frac{1}{4}\left(\left\|u_{t}\right\|^{2}+\left\|u_{x t}\right\|^{2}+\left\|u_{x x}\right\|^{2}\right) \\
& =\frac{1}{4}\left\|\left(u(t), u_{t}(t)\right)\right\|_{V \times U}^{2} .
\end{aligned}
$$

Thus, $\widetilde{E}(t)$ dominates $\left\|\left(u(t), u_{t}(t)\right)\right\|_{V \times U}$. Also, from (45), we have

$$
\begin{aligned}
\left|\widetilde{E}_{\varepsilon}(t)-\widetilde{E}(t)\right| & =|\varepsilon \varphi(t)| \\
& =\left|\varepsilon \int_{0}^{L}\left(u_{t}-u_{x x t}\right) u d x\right| \\
& \leq \frac{\varepsilon\left(L^{2}+L^{4}\right)}{2}\left(\left\|u_{t}\right\|^{2}+\left\|u_{x t}\right\|^{2}+\left\|u_{x x}\right\|^{2}\right) \\
& \leq 2 \varepsilon\left(L^{2}+L^{4}\right) \widetilde{E}(t)
\end{aligned}
$$

which implies that, for $\varepsilon$ sufficiently small enough,

$$
\begin{aligned}
\left(1-2 \varepsilon\left(L^{2}+L^{4}\right)\right) \widetilde{E}(t) & \leq \widetilde{E}_{\varepsilon}(t) \\
& \leq\left(1+2 \varepsilon\left(L^{2}+L^{4}\right)\right) \widetilde{E}(t) .
\end{aligned}
$$

Inserting (66) into (62), we get

$$
\begin{aligned}
& \frac{d}{d t} \widetilde{E}_{\varepsilon}(t)+\left(\frac{1}{1+2 \varepsilon\left(L^{2}+L^{4}\right)}\right) \widetilde{E}_{\varepsilon}(t) \\
& \quad \leq \varepsilon\left(L_{0}+L_{1}+L^{4}\|q\|^{2}\right) .
\end{aligned}
$$

Applying Gronwall's inequality, we obtain

$$
\begin{aligned}
\widetilde{E}_{\varepsilon}(t) \leq & \widetilde{E}_{\varepsilon}(0) \exp \left(-\frac{1}{1+2 \varepsilon\left(L^{2}+L^{4}\right)} t\right) \\
= & \left|\varepsilon \int_{0}^{L}\left(u_{t}-u_{x x t}\right) u d x\right| \\
& +\varepsilon\left(L_{0}+L_{1}+L^{4}\|q\|^{2}\right)\left(1+2 \varepsilon\left(L^{2}+L^{4}\right)\right) \\
& \cdot\left(1-\exp \left(-\frac{1}{1+2 \varepsilon\left(L^{2}+L^{4}\right)} t\right)\right) .
\end{aligned}
$$

Since the given invariant set $B$ is bounded, $\widetilde{E}_{\varepsilon}(0)$ is also bounded. Then there exists $t_{B}>0$ large enough such that

$$
\begin{array}{r}
{\left[1-2 \varepsilon\left(L^{2}+L^{4}\right)\right] \widetilde{E}(t)} \\
\leq \widetilde{E}_{\varepsilon}(t) \leq \varepsilon\left(L_{0}+L_{1}+L^{4}\|q\|^{2}\right)\left(1+2 \varepsilon\left(L^{2}+L^{4}\right)\right), \\
\forall t>t_{B} .
\end{array}
$$


Then from (64) we have

$$
\begin{array}{r}
\left\|\left(u(t), u_{t}(t)\right)\right\|_{V \times U}^{2} \\
\leq \frac{4 \varepsilon\left(L_{0}+L_{1}+L^{4}\|q\|^{2}\right)\left(1+2 \varepsilon\left(L^{2}+L^{4}\right)\right)}{1-2 \varepsilon\left(L^{2}+L^{4}\right)}, \\
\forall t>t_{B} .
\end{array}
$$

This shows that

$$
\begin{aligned}
& \mathbb{B}=\left\{\left(u, u_{t}\right) \in H_{0}:\right. \\
& \left\|\left(u, u_{t}\right)\right\|_{H_{0}}^{2} \\
& \left.\leq \frac{4 \varepsilon\left(L_{0}+L_{1}+L^{4}\|q\|^{2}\right)\left(1+2 \varepsilon\left(L^{2}+L^{4}\right)\right)}{1-2 \varepsilon\left(L^{2}+L^{4}\right)}\right\}
\end{aligned}
$$

is an absorbing set for $S(t)$ in $H_{0}$. The proof of Theorem 4 is ended.

A semigroup $S(t)$ is asymptotically smooth in $H$ if for any bounded positive invariant set $B \subset H$, there exists a compact set $K \subset \bar{B}$ such that

$$
\operatorname{dist}(S(t) B, K) \longrightarrow 0 \quad \text { as } t \longrightarrow \infty \text {. }
$$

Then the following lemma is well known.

Lemma 5 (see [16], Theorem 2.3). Let $S(t)$ be a dissipative $C_{0^{-}}$ semigroup defined on a metric space $H$; then $S(t)$ has a compact global attractor in $\mathrm{H}$ if and only if it is asymptotically smooth in $\mathrm{H}$.

The asymptotic smoothness can be verified from a result by Khanmamedov [17] and Chueshov and Lasiecka [16]. Assume that $H$ is a Banach space.

Lemma 6 (see [16], Proposition 2.10). Assume that for any bounded positive invariant set $B \subset H$ and for any $\varepsilon>0$, there exists $T=T(\varepsilon, B)$ such that

$$
\|S(T) x-S(T) y\| \leq \varepsilon+\phi_{T}(x, y), \quad \forall x, y \in B,
$$

where $\phi_{T}: H \times H \rightarrow R$ satisfies

$$
\liminf _{k \rightarrow \infty} \liminf _{l \rightarrow \infty} \phi_{T}\left(z_{k}, z_{l}\right)=0
$$

for any sequence $\left(z_{n}\right)$ of $B$. Then $S(t)$ is asymptotically smooth in $\mathrm{H}$.

Theorem 7. Assume the hypotheses of Theorem 2 and $\varrho=r=$ 0 ; then the corresponding semigroup $S(t)$ of problem (1)-(4) is asymptotic compactness.

Proof. We are going to apply Lemma 6 to prove the asymptotic compactness. Given initial data $\left(u^{0}, u^{1}\right)$ and $\left(v^{0}, v^{1}\right)$ in a bounded invariant set $B \subset H_{0}$, let $u, v$ be the corresponding weak solutions of problem (1)-(4). Then the difference $w=$ $u-v$ is a weak solution of

$$
\begin{gathered}
w_{t t}-w_{x x t t}+w_{x x x x}-\sigma\left(\left\|u_{x}\right\|^{2}\right) w_{x x}-\Delta \sigma v_{x x} \\
-\phi\left(\left\|u_{x}\right\|^{2}\right) w_{x x t}-\Delta \phi v_{x x t}=0 \\
w(0, t)=w_{x}(L \cdot t)=w_{x x}(0, t)=0, \\
w_{x x x}(L, t)=\Delta f+\Delta g
\end{gathered}
$$

where

$$
\begin{gathered}
\Delta \sigma=\sigma\left(\left\|u_{x}\right\|^{2}\right)-\sigma\left(\left\|v_{x}\right\|^{2}\right), \\
\Delta \phi=\phi\left(\left\|u_{x}\right\|^{2}\right)-\phi\left(\left\|v_{x}\right\|^{2}\right), \\
\Delta f=f(u(L, t))-f(v(L, t)), \\
\Delta g=g\left(u_{t}(L, t)\right)-g\left(v_{t}(L, t)\right) .
\end{gathered}
$$

Let us assume

$$
F(t)=\frac{1}{2}\left\|w_{t}\right\|^{2}+\frac{1}{2}\left\|w_{x t}\right\|^{2}+\frac{1}{2}\left\|w_{x x}\right\|^{2}+\frac{1}{2} \sigma\left(\left\|u_{x}\right\|^{2}\right)\left\|w_{x}\right\|^{2}
$$

and define

$$
\psi(t)=\int_{0}^{L}\left(w_{t}-w_{x x t}\right) w d x
$$

As before, by density, we can assume formally that $w$ is sufficiently regular. Then, multiplying the first equation in (75) by $w_{t}$ and integrating over $(0, L)$, we get

$$
\begin{aligned}
& \frac{1}{2} \frac{d}{d t}\left(\left\|w_{t}\right\|^{2}+\left\|w_{x t}\right\|^{2}+\left\|w_{x x}\right\|^{2}+\sigma\left(\left\|u_{x}\right\|^{2}\right)\left\|w_{x}\right\|^{2}\right) \\
& \quad+\phi\left(\left\|u_{x}\right\|^{2}\right)\left\|w_{x t}\right\|^{2}+w_{x x x}(L, t) w_{t}(L, t) \\
& =\sigma^{\prime}\left(\left\|u_{x}\right\|^{2}\right) \int_{0}^{L} u_{x} u_{x t} d x\left\|w_{x}\right\|^{2}+\Delta \sigma \int_{0}^{L} v_{x x} w_{t} d x \\
& \quad+\Delta \phi \int_{0}^{L} v_{x x t} w_{t} d x .
\end{aligned}
$$

Taking into account the third equation in (75) we see that

$$
\begin{aligned}
& \frac{d}{d t} F(t)+\phi\left(\left\|u_{x}\right\|^{2}\right)\left\|w_{x t}\right\|^{2}+\Delta g w_{t}(L, t) \\
& =\sigma^{\prime}\left(\left\|u_{x}\right\|^{2}\right) \int_{0}^{L} u_{x} u_{x t} d x\left\|w_{x}\right\|^{2}+\Delta \sigma \int_{0}^{L} v_{x x} w_{t} d x \\
& \quad+\Delta \phi \int_{0}^{L} v_{x x t} w_{t} d x-\Delta f w_{t}(L, t)
\end{aligned}
$$


By multiplying first equation in (75) by $w$ and integrating over $(0, L)$, we obtain that

$$
\begin{aligned}
& \frac{d}{d t} \phi(t)-\left\|w_{t}\right\|^{2}-\left\|w_{x t}\right\|^{2}+\left\|w_{x x}\right\|^{2} \\
& +\sigma\left(\left\|u_{x}\right\|^{2}\right)\left\|w_{x}\right\|^{2} \\
& +\phi\left(\left\|u_{x}\right\|^{2}\right) \int_{0}^{L} w_{x} w_{x t} d x+w_{x x x} w(L, t) \\
& \quad-\Delta \sigma \int_{0}^{L} v_{x x} w d x-\Delta \phi \int_{0}^{L} v_{x x t} w d x=0 .
\end{aligned}
$$

Also taking into account the third equation in (75) we see that

$$
\begin{aligned}
& \frac{d}{d t} \phi(t)+2 F(t) \\
& =-\phi\left(\left\|u_{x}\right\|^{2}\right) \int_{0}^{L} w_{x} w_{x t} d x-(\Delta f+\Delta g) w(L, t) \\
& \quad+\Delta \sigma \int_{0}^{L} v_{x x} w d x \\
& \quad-\Delta \phi \int_{0}^{L} v_{x t} w_{x} d x+2\left\|w_{t}\right\|^{2}+2\left\|w_{x t}\right\|^{2} .
\end{aligned}
$$

Then summing (80) with $\eta$ times (82) we obtain that

$$
\begin{aligned}
& \frac{d}{d t} F(t)+\eta \frac{d}{d t} \phi(t)+\eta F(t)+\eta F(t) \\
& \quad+\phi\left(\left\|u_{x}\right\|^{2}\right)\left\|w_{x t}\right\|^{2}+\Delta g w_{t}(L, t) \\
& =\sigma^{\prime}\left(\left\|u_{x}\right\|^{2}\right) \int_{0}^{L} u_{x} u_{x t} d x\left\|w_{x}\right\|^{2}+\Delta \sigma \int_{0}^{L} v_{x x} w_{t} d x \\
& \quad+\Delta \phi \int_{0}^{L} v_{x x t} w_{t} d x-\Delta f w_{t}(L, t) \\
& \quad-\eta \phi\left(\left\|u_{x}\right\|^{2}\right) \int_{0}^{L} w_{x} w_{x t} d x-\eta(\Delta f+\Delta g) w(L, t) \\
& \quad+\eta \Delta \sigma \int_{0}^{L} v_{x x} w d x \\
& \quad-\eta \Delta \phi \int_{0}^{L} v_{x t} w_{x} d x+2 \eta\left\|w_{t}\right\|^{2}+2 \eta\left\|w_{x t}\right\|^{2} .
\end{aligned}
$$

In view of assumption (17) of the function $g$, we obtain that

$$
\Delta g w_{t}(L, t) \geq k_{1}\left|w_{t}(L, t)\right|^{2} .
$$

Also since $\phi(s) \geq \alpha+\beta s^{\gamma}(\alpha, \beta>0, \gamma \geq 1)$, we see that

$$
\phi\left(\left\|u_{x}\right\|^{2}\right)\left\|w_{x t}\right\|^{2} \geq\left(\alpha+\beta\left\|u_{x}\right\|^{2 \gamma}\right)\left\|w_{x t}\right\|^{2} .
$$

In the following, let us estimate the right hand side of (83). We recall that $u, v$, and $w$ satisfy the estimate $\left\|u_{t}\right\|^{2}+\left\|u_{x t}\right\|^{2}+$ $\left\|u_{x x}\right\|^{2}+\widehat{\sigma}\left(\left\|u_{x}\right\|^{2}\right) \leq M_{1}$, then denoting by $C_{0}$ a generic positive constant which depends only on $B$ we can simplify several notations.
Firstly, since $\sigma(\cdot), \phi(\cdot) \in C^{1}(R), \phi(0)=0$, and $\left\|u_{x}\right\|^{2} \leq C_{0}$, we get $\sigma^{\prime}\left(\left\|u_{x}\right\|^{2}\right) \leq C_{0}, \phi\left(\left\|u_{x}\right\|^{2}\right) \leq C_{0}$; then we have

$$
\begin{aligned}
& \sigma^{\prime}\left(\left\|u_{x}\right\|^{2}\right) \int_{0}^{L} u_{x} u_{x t} d x\left\|w_{x}\right\|^{2} \leq C_{0}\left\|w_{x}\right\|^{2} ; \\
& -\eta \phi\left(\left\|u_{x}\right\|^{2}\right) \int_{0}^{L} w_{x} w_{x t} d x \leq C_{0}\left\|w_{x}\right\|\left\|w_{x t}\right\| .
\end{aligned}
$$

From the mean value theorem and noting that $\sigma\left(a^{2}\right)-$ $\sigma\left(b^{2}\right) \leq \sigma^{\prime}\left(\sup \left\{a^{2}, b^{2}\right\}\right)|a+b||a-b|$ and $\phi\left(a^{2}\right)-\phi\left(b^{2}\right) \leq$ $\phi^{\prime}\left(\sup \left\{a^{2}, b^{2}\right\}\right)|a+b||a-b|$, we have

$$
\begin{gathered}
\Delta \sigma \int_{0}^{L} v_{x x} w_{t} d x \leq C_{0}\left\|w_{x}\right\|\left\|w_{t}\right\|, \\
\eta \Delta \sigma \int_{0}^{L} v_{x x} w d x \leq C_{0}\left\|w_{x}\right\|^{2}, \\
\Delta \phi \int_{0}^{L} v_{x x t} w_{t} d x \leq C_{0}\left\|w_{x}\right\|\left\|w_{x t}\right\|, \\
-\eta \Delta \phi \int_{0}^{L} v_{x t} w_{x} d x \leq C_{0}\left\|w_{x}\right\|^{2} .
\end{gathered}
$$

From assumption (19) of the function $f$ and $\varrho=0$ and inequalities (44), we get

$$
\begin{aligned}
\left|-\Delta f w_{t}(L)\right| & \leq 3 k\|w(t)\|_{\infty}\left|w_{t}(l, t)\right| \\
& \leq C_{0}\left\|w_{x}\right\|\left|w_{t}(L)\right| \\
& \leq C_{0}\left\|w_{x}\right\|^{2}+\frac{k}{2}\left|w_{t}(L)\right|^{2}, \\
|-\eta w(L) \Delta f| & \leq \eta 3 k\|w\|_{\infty}|w(L)| \\
& \leq \eta 3 k\|w\|_{\infty} \sqrt{L}\left\|w_{x}\right\| \\
& \leq C_{0}\left\|w_{x}\right\|^{2} .
\end{aligned}
$$

From assumption (18) of the function $g$ and $r=0$ and inequalities (44), we get

$$
\begin{aligned}
|-\eta w(L, t) \Delta g| & \leq \eta 3 k_{2}|w(L, t)|\left|w_{t}(L, t)\right| \\
& \leq \eta \sqrt{L}\left\|w_{x}\right\| 3 k_{2}\left|w_{t}(L, t)\right| \\
& \leq \frac{9 \eta^{2} L k_{2}^{2}}{2 k_{1}}\left\|w_{x}\right\|^{2}+\frac{k_{1}}{2}\left|w_{t}(L, t)\right|^{2} .
\end{aligned}
$$

Substituting (84)-(89) into (83) and using Schwarz inequality, we obtain that

$$
\begin{aligned}
& \frac{d}{d t} F(t)+\eta \frac{d}{d t} \phi(t)+2 \eta F(t)+\left(\alpha+\beta\left\|u_{x}\right\|^{\gamma}\right)\left\|w_{x t}\right\|^{2} \\
& \quad \leq\left(5 C_{0}+\frac{9 \eta^{2} L k_{2}^{2}}{2 k_{1}}+\frac{2 C_{0}^{2}}{\eta}\right)\left\|w_{x}\right\|^{2}+(3 \eta L+3 \eta)\left\|w_{x t}\right\|^{2}
\end{aligned}
$$


With $\eta \leq \alpha /(3 L+3)$ sufficiently small enough, we have

$$
\begin{aligned}
& \frac{d}{d t} F(t)+\eta \frac{d}{d t} \phi(t)+2 \eta F(t) \\
& \quad \leq\left(5 C_{0}+\frac{9 \eta^{2} L k_{2}^{2}}{2 k_{1}}+\frac{2 C_{0}^{2}}{\eta}\right)\left\|w_{x}\right\|^{2} .
\end{aligned}
$$

Defining $F_{\eta}(t)=F(t)+\eta \phi(t)$ and considering (44)-(45), we have

$$
\begin{aligned}
\left|F_{\eta}(t)-F(t)\right| & =|\eta \phi(t)| \\
& =\left|\eta \int_{0}^{L}\left(w_{t}-w_{x x t}\right) w d x\right| \\
& \leq \eta\left\|w_{t}\right\|\|w\|+\eta\left\|w_{x t}\right\|\left\|w_{x}\right\| \\
& \leq \eta\left\|w_{t}\right\| L^{2}\left\|w_{x x}\right\|+\eta\left\|w_{x t}\right\| \sqrt{L}\left\|w_{x x}\right\| \\
& \leq \eta L^{2}\left(\left\|w_{t}\right\|^{2}+\left\|w_{x t}\right\|^{2}+\left\|w_{x x}\right\|^{2}\right) \\
& \leq \eta L^{2} F(t),
\end{aligned}
$$

which implies for $\eta<\min \left\{1 / L^{2}, \alpha /(3 L+3)\right\}$ sufficiently small enough that

$$
\left(1-\eta L^{2}\right) F(t) \leq F_{\eta}(t) \leq\left(1+\eta L^{2}\right) F(t) .
$$

Let $C_{1}=5 C_{0}+9 \eta^{2} L k_{2}^{2} / 2 k_{1}+2 C_{0}^{2} / \eta$; then inserting (93) into (91), we obtain that

$$
\frac{d}{d t} F_{\eta}(t)+\frac{2 \eta}{1+\eta L^{2}} F_{\eta}(t) \leq C_{1}\left\|w_{x}\right\|^{2}, \quad t \geq 0 .
$$

From Gronwall's lemma, we get

$$
\begin{aligned}
F_{\eta}(t) \leq & F_{\eta}(0) e^{-\left(2 \eta /\left(1+\eta L^{2}\right)\right) t} \\
& +C_{1} \int_{0}^{t} e^{-\left(\eta /\left(1+\eta L^{2}\right)\right)(t-s)}\left\|w_{x}(s)\right\|^{2} d s .
\end{aligned}
$$

On the other hand, we have

$$
\begin{aligned}
F_{\eta}(t) \geq & \left(1-\eta L^{2}\right) F(t) \\
= & \frac{\left(1-\eta L^{2}\right)}{2}\left(\left\|w_{t}\right\|^{2}+\left\|w_{x t}\right\|^{2}+\left\|w_{x x}\right\|^{2}\right. \\
& \left.+\sigma\left(\left\|u_{x}\right\|^{2}\right)\left\|w_{x}\right\|^{2}\right) \\
\geq & \frac{1-\eta L^{2}}{2}\left(\left\|w_{t}\right\|^{2}+\left\|w_{x t}\right\|^{2}+\left\|w_{x x}\right\|^{2}\right) .
\end{aligned}
$$

Therefore, combining (95) and (96), we can fix a constant $C_{B}>0$, depending on the size of $B$ but not on $t>0$, such that $E_{1}(0) \leq C_{B}$; so

$$
\begin{aligned}
\left\|\left(w(t), w_{t}(t)\right)\right\|_{V \times U} \leq & \frac{\sqrt{2}}{\sqrt{1-\eta L^{2}}} C_{B} \exp \left(-\frac{\eta}{1+\eta L^{2}} t\right) \\
& +\sqrt{\frac{2 C_{1}}{1-\eta L^{2}}}\left[\int_{0}^{t}\left\|w_{x}(s)\right\|^{2} d s\right]^{1 / 2} .
\end{aligned}
$$

Given $\varepsilon>0$, we choose $T$ large such that

$$
\sqrt{\frac{2}{1-\eta L^{2}}} C_{B} \exp \left(-\frac{\eta}{1+\eta L^{2}} t\right) \leq \varepsilon
$$

and define $\phi_{T}: H_{0} \times H_{0} \rightarrow R$ as

$$
\begin{aligned}
\phi_{T} & \left(\left(u^{0}, u^{1}\right),\left(v^{0}, v^{1}\right)\right) \\
& =\sqrt{\frac{2 C_{1}}{1-\eta L^{2}}}\left[\int_{0}^{T}\left\|u_{x}(t)-v_{x}(t)\right\|^{2} d t\right]^{1 / 2} .
\end{aligned}
$$

Then from (97)-(99), we get

$$
\begin{gathered}
\left\|S(T)\left(u^{0}, u^{1}\right)-S(T)\left(v^{0}, v^{1}\right)\right\|_{H_{0}} \\
\leq \varepsilon+\phi_{T}\left(\left(u^{0}, u^{1}\right),\left(v^{0}, v^{1}\right)\right)
\end{gathered}
$$

for all $\left(u^{0}, u^{1}\right),\left(v^{0}, v^{1}\right) \in B$

Let $\left(u_{n}^{0}, u_{n}^{1}\right)$ be a given sequence of initial data in $B$. Then the corresponding sequence $\left(u_{n}(t), u_{t n}\right)$ of solutions of problem (1)-(4) is uniformly bounded in $H_{0}$, because $B$ is bounded and positively invariant. So $\left\{u_{n}\right\}$ is bounded in $C([0, \infty), V) \cap C^{1}([0, \infty), U)$. Since $V \hookrightarrow H_{0}^{1}(\Omega)$ compactly, there exists a subsequence $u_{n k}$ which converges strongly in $C\left([0, T], H_{0}^{1}(\Omega)\right)$. Therefore

$$
\begin{aligned}
& \lim _{k \rightarrow \infty} \lim _{l \rightarrow \infty} \int_{0}^{T}\left\|u_{x n k}(s)-u_{x n l}(s)\right\|^{2} d s=0, \\
& \lim _{k \rightarrow \infty} \lim _{l \rightarrow \infty} \phi_{T}\left(\left(u_{n k}^{0}, u_{n k}^{1}\right),\left(u_{n l}^{0}, u_{n l}^{1}\right)\right)=0 .
\end{aligned}
$$

So $S(t)$ is asymptotically smooth in $H_{0}$. That is, Lemma 6 holds. Thus Theorem 7 is proved.

Remark 8. The novelty and difficulty of Theorems 4 lie in the appropriate definition on the functions $\varphi(t)=\int_{0}^{L}\left(u_{t}-\right.$ $\left.u_{x x t}\right) u d x, \psi(t)=\int_{0}^{L}\left(w_{t}-w_{x x t}\right) w d x$ and the relationship between $\widetilde{E}_{\varepsilon}(t)$ and the energy function $\widetilde{E}(t)$ and the relationship between $F_{\eta}(t)$ and the function $F(t)$.

Lemma 9 ([18], Theorem 1.1 in Chapter I). One assumes that $H$ is a metric space and that the operators $\{S(t)\}_{t \geq 0}$ are given and satisfy the usual group properties and $S(t)$ is continuous operator from $H$ into itself and the semigroup $\{S(t)\}_{t \geq 0}$ is asymptotically compact. One also assumes that there exist an open set $U$ and a bounded set $\mathbb{B}$ of $U$ such that $U$ is absorbing in $\mathbb{B}$. Then $\omega(\mathbb{B})$ is a compact attractor.

By view of Lemma 9, with Theorems 4 and 7, the main result of a global attractor reads as follows.

Theorem 10. The corresponding semigroup $S(t)$ of problem (1)-(4) has a compact global attractor in the phase space $H_{0}$.

\section{Conflict of Interests}

The authors declare that there is no conflict of interests regarding the publication of this paper. 


\section{Acknowledgments}

The project is supported by the National Natural Science Foundation of China (Grant no. 11172194 and no. 11401420), the Natural Science Foundation of Shanxi Province, China (Grant no. 2010011008), and the Natural Science Foundation for Young Scientists of Shanxi Province, China (Grant no. 2011021002-2).

\section{References}

[1] S. Woinowsky-Krieger, "The effect of axial force on the vibration of hinged bars," Journal of Applied Mechanics, vol. 17, pp. 35-36, 1950.

[2] J. M. Ball, "Initial-boundary value problems for an extensible beam," Journal of Mathematical Analysis and Applications, vol. 42, pp. 61-90, 1973.

[3] L. A. Medeiros, "On a new class of nonlinear wave equations," Journal of Mathematical Analysis and Applications, vol. 69, no. 1, pp. 252-262, 1979.

[4] S. K. Patcheu, "On a global solution and asymptotic behaviour for the generalized damped extensible beam equation," Journal of Differential Equations, vol. 135, no. 2, pp. 299-314, 1997.

[5] T. F. Ma and V. Narciso, "Global attractor for a model of extensible beam with nonlinear damping and source terms," Nonlinear Analysis. Theory, Methods \& Applications, vol. 73, no. 10, pp. 3402-3412, 2010.

[6] C. Giorgi, M. G. Naso, V. Pata, and M. Potomkin, "Global attractors for the extensible thermoelastic beam system," Journal of Differential Equations, vol. 246, no. 9, pp. 3496-3517, 2009.

[7] Z. J. Yang, "On an extensible beam equation with nonlinear damping and source terms," Journal of Differential Equations, vol. 254, no. 9, pp. 3903-3927, 2013.

[8] A. F. Pazoto and G. P. Menzala, "Uniform stabilization of a nonlinear beam model with thermal effects and nonlinear boundary dissipation," Funkcialaj Ekvacioj, vol. 43, no. 2, pp. 339-360, 2000.

[9] T. F. Ma, "Boundary stabilization for a non-linear beam on elastic bearings," Mathematical Methods in the Applied Sciences, vol. 24, no. 8, pp. 583-594, 2001.

[10] T. F. Ma, V. Narciso, and M. L. Pelicer, "Long-time behavior of a model of extensible beams with nonlinear boundary dissipations," Journal of Mathematical Analysis and Applications, vol. 396, no. 2, pp. 694-703, 2012.

[11] P. G. Geredeli and I. Lasiecka, "Asymptotic analysis and upper semicontinuity with respect to rotational inertia of attractors to von Karman plates with geometrically localized dissipation and critical nonlinearity," Nonlinear Analysis :Theory, Methods \& Applications, vol. 91, pp. 72-92, 2013.

[12] G. Ji and I. Lasiecka, "Nonlinear boundary feedback stabilization for a semilinear Kirchhoff plate with dissipation acting only via moments-limiting behavior," Journal of Mathematical Analysis and Applications, vol. 229, no. 2, pp. 452-479, 1999.

[13] I. Chueshov, "Global attractors for a class of Kirchhoff wave models with a structural nonlinear damping," Journal of Abstract Differential Equations and Applications, vol. 1, no. 1, pp. 86-106, 2010.

[14] I. Chueshov, "Long-time dynamics of Kirchhoff wave models with strong nonlinear damping," Journal of Differential Equations, vol. 252, no. 2, pp. 1229-1262, 2012.
[15] Z. Yang, P. Ding, and Z. Liu, "Global attractor for the Kirchhoff type equations with strong nonlinear damping and supercritical nonlinearity," Applied Mathematics Letters, vol. 33, pp. 12-17, 2014.

[16] I. Chueshov and I. Lasiecka, "Long-time behavior of second order evolution equations with nonlinear damping," Memoirs of the American Mathematical Society, vol. 195, no. 912, 2008.

[17] A. K. Khanmamedov, "Global attractors for von Karman equations with nonlinear interior dissipation," Journal of Mathematical Analysis and Applications, vol. 318, no. 1, pp. 92-101, 2006.

[18] R. Teman, Infinite Dimensional Dynamic System in Mechanics and Physics, Springer, New York, NY, USA, 1998. 


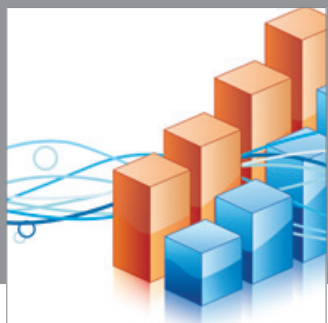

Advances in

Operations Research

mansans

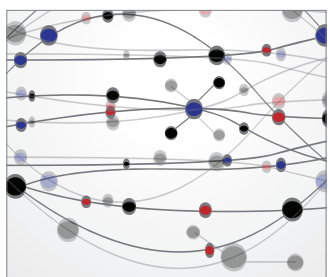

The Scientific World Journal
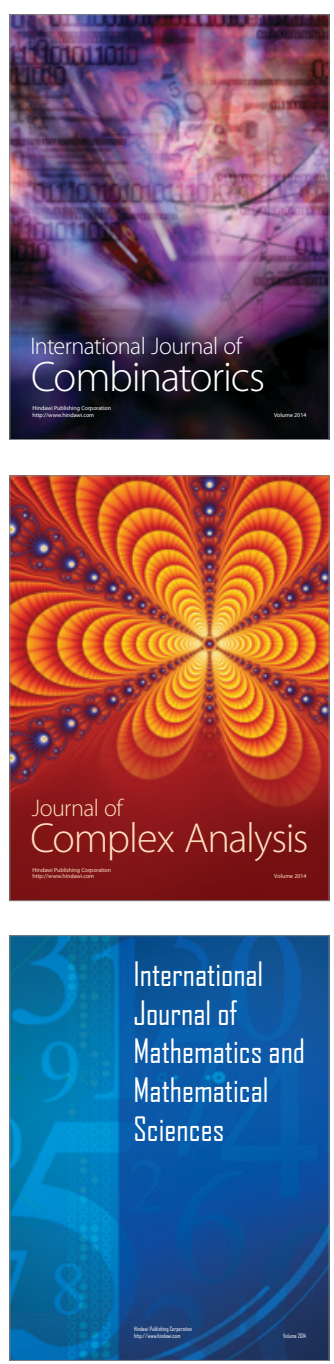
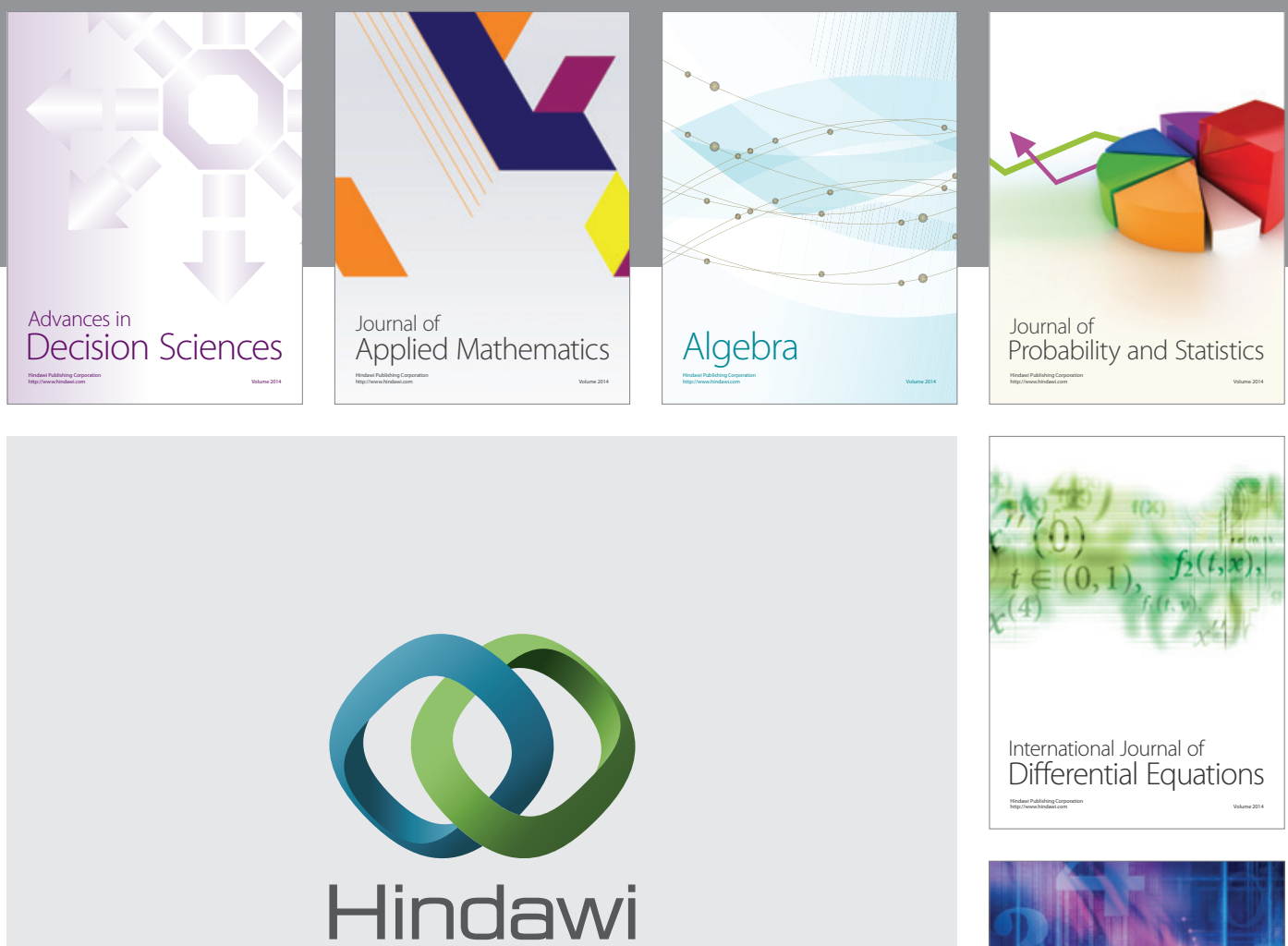

Submit your manuscripts at http://www.hindawi.com
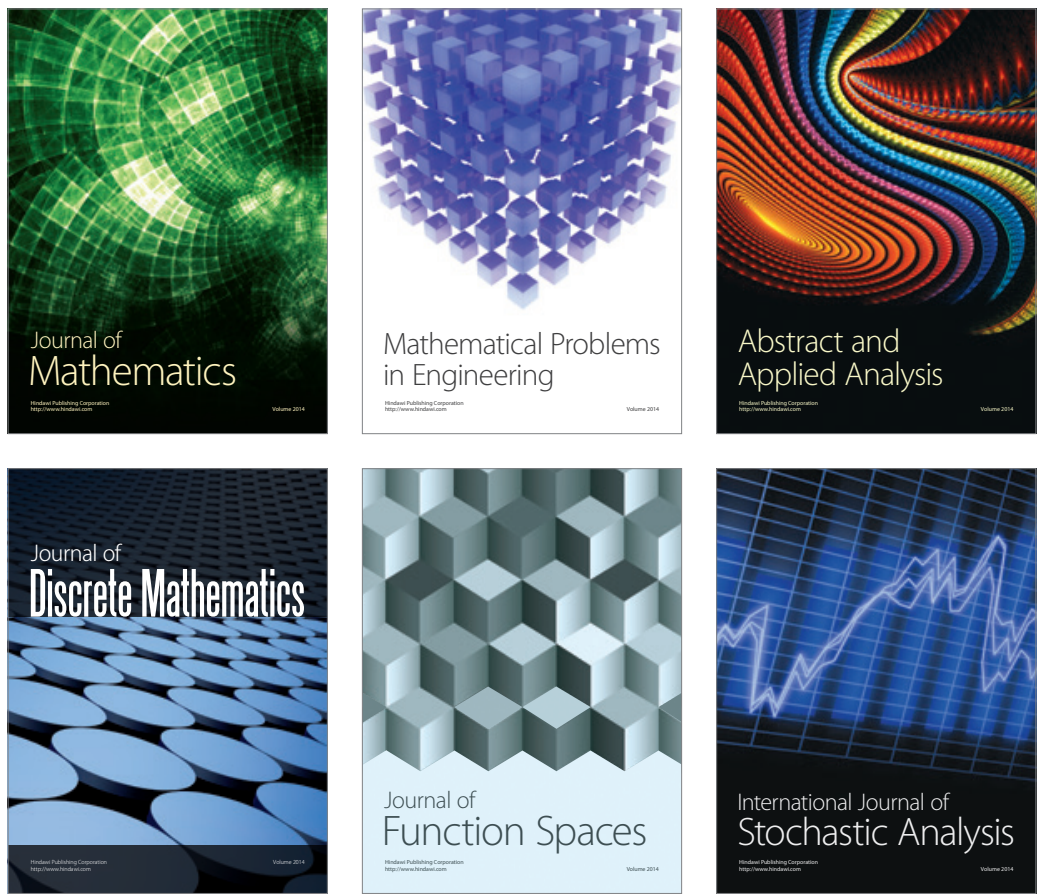

Journal of

Function Spaces

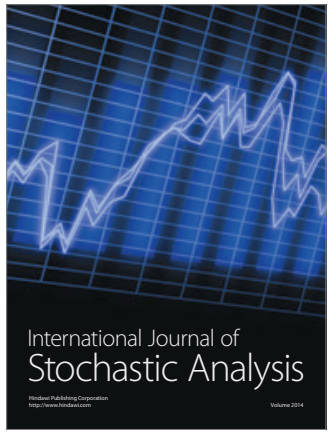

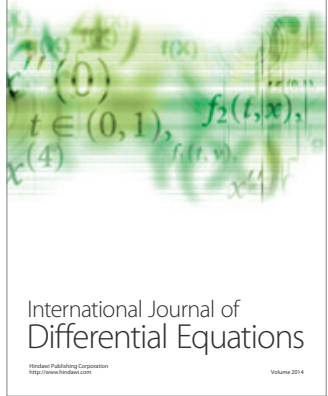
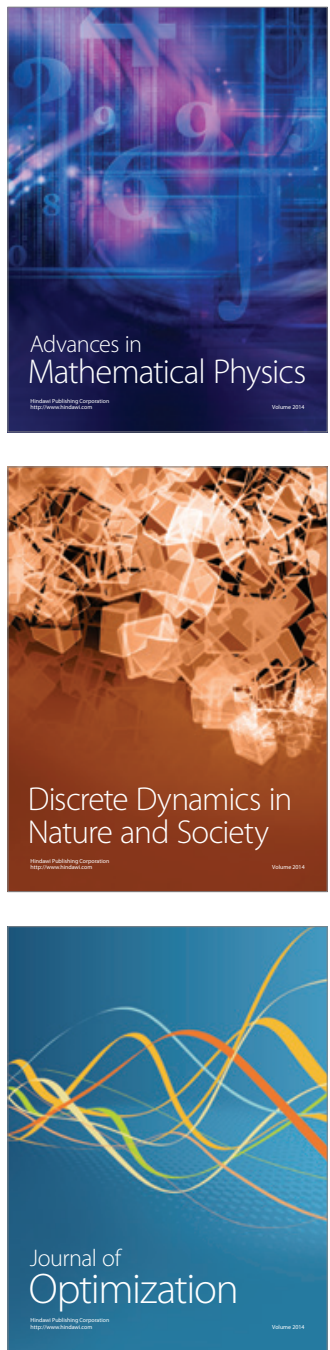